\title{
Hypothermia due to limbic system involvement and longitudinal myelitis in a case of Japanese encephalitis: a case report from India
}

This article was published in the following Dove Press journal:

International Journal of General Medicine

12 April 2017

Number of times this article has been viewed

\author{
Santhosh Narayanan' \\ NK Thulaseedharan' \\ Gomathy Subramaniam² \\ Geetha Panarkandy' \\ VK Shameer' \\ Arathi Narayanan' \\ 'Department of General Medicine, \\ ${ }^{2}$ Department of Radiodiagnosis, \\ Government Medical College, \\ Kozhikode, Kerala, India
}

\begin{abstract}
Japanese encephalitis (JE) is an infectious encephalitis prevalent in Asia. It usually presents with fever, headache, convulsions and extrapyramidal symptoms. Limbic system involvement and hypothermia though common in autoimmune encephalitis have never been reported in JE. We report a case of an 18-year-old girl with no previous comorbidities who presented to us with a history of fever and headache for 1 week duration. She developed bilateral lateral rectus palsy and asymmetric flaccid weakness of all four limbs, after 2 days of admission, which was followed by altered sensorium and intermittent hypothermia. Neuroimaging revealed longitudinal myelitis extending from pons till the L1 level along with bilateral thalamic hemorrhage in brain. Cerebrospinal fluid (CSF) was positive for IgM antibody to JE virus. She was treated with supportive measures, but she developed intractable hypothermia and seizures and succumbed to illness after 2 weeks of admission.
\end{abstract}

Keywords: Japanese encephalitis, hypothermia, limbic system

\section{Introduction}

Japanese encephalitis (JE) is a vector-borne disease of the tropics spread by Culex mosquitoes and maintained in the environment by amplifier hosts. Human beings are incidental dead-end hosts. Although very few cases are symptomatic, it causes serious neuroinvasive disease leading to either mortality or neurological sequelae in survivors. Here, we describe a case of an 18-year-old girl infected with JE complicated by bilateral lateral rectus palsy due to pontine involvement, longitudinal myelitis and hypothermia.

\section{Case report}

An 18-year-old girl hailing from Kozhikode, South India, with no past history of any illness, visited our hospital with complaints of fever of 1 week duration. She also had headache that was increasing in intensity and aggravated in the early morning. She also had two episodes of vomiting. She complained of double vision on the third day of admission, which was followed by asymmetric weakness of both proximal distal muscles of all four limbs. There was no history of sensory disturbances and bladder or bowel dysfunction. On examination, her vitals were stable. There was no pallor, icterus, clubbing, lymphadenopathy, rashes or eschar on general examination. Neurological examination on admission revealed normal speech, intelligence and memory. There was no papilledema. She developed bilateral lateral rectus palsy (Figure 1A and B), with normal upgaze and papillary reflexes. Other cranial nerves were within normal limits. Motor system examination showed normal muscle bulk. Muscle groups in all
Correspondence: Santhosh Narayanan Department of General Medicine, Government Medical College, Kozhikode, Kerala 673008, India

Tel +9l 9895658833

Email drsanthosh4@gmail.com 
limbs showed hypotonia with grade $3 / 5$ power in the right lower limb, grade $2 / 5$ power in the left lower limb and grade $4 / 5$ power in both upper limbs. All deep tendon reflexes were sluggish. Sensory system examination was normal. There was neck stiffness with other signs of meningeal irritation. Rest of the systems were within normal limits. Her clinical course is summarized in Figure 2.

Initial investigations revealed normal hemogram, with an elevated erythrocyte sedimentation rate (ESR) of $95 \mathrm{~mm}$ in the first hour. Blood sugar, serum electrolytes and renal and liver function tests were within normal limits. Computerized tomography of brain was within normal limits.

With a history of fever and headache and findings of meningitis, lumbar puncture was done, which showed an opening pressure of $190 \mathrm{~mm}$ of cerebrospinal fluid (CSF). CSF analysis revealed a CSF total count of 30 cells with $80 \%$ lymphocytes. CSF sugar was normal, and protein was mildly elevated $(60 \mathrm{mg} /$ dL [normal 20-40 mg/dL]). Bacterial cultures were sterile. CSF adenosine deaminase and culture for Mycobacterium tuberculosis were negative. India ink stain for cryptococci was negative. Cytology did not show any malignant cells. CSF viral serology turned out to be positive for JE virus with positive IgM antibodies specific for the same insignificant titer. Autoimmune markers in CSF (NMDAR, LGI1, Caspr2, AMPAR [GluR1 and GluR2 subunits] and GABA-B-R) were negative.

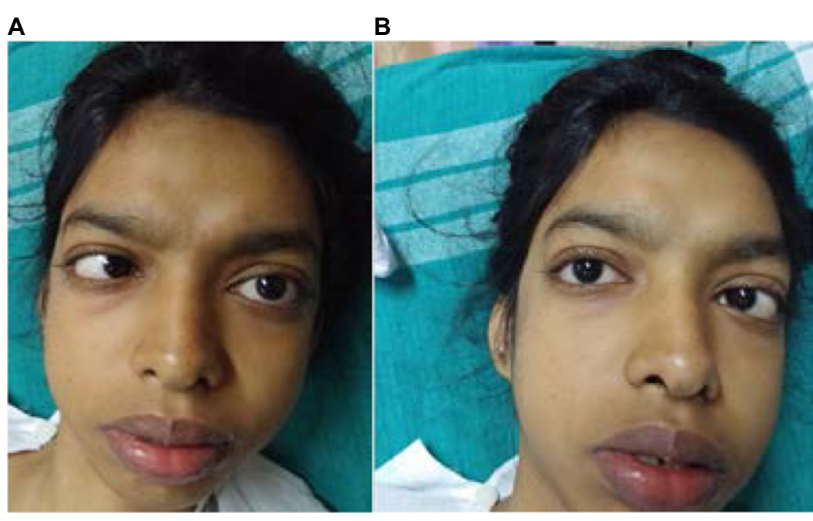

Figure I (A and B) Bilateral lateral rectus palsy.
Magnetic resonance imaging (MRI) of the brain showed hyperintensities in bilateral thalami and in the limbic region, suggestive of limbic encephalitis (Figure 3). MRI of the spine showed T2-weighted hyperintense signal intensities in the spinal cord caudally till the L1 level, predominantly involving the anterior horn cells, and rostrally, they extended upto pons, suggestive of longitudinal myelitis (Figure 4). Electroencephalogram displayed slowing of delta waves in the frontotemporal region.

The patient was treated with supportive measures. However, she deteriorated with the development of altered sensorium and intermittent hypothermia. Oral, axillary, rectal temperature revealed a temperature $<94^{\circ} \mathrm{F}$ on multiple occasions. Electrocardiogram at the time of hypothermia showed bradycardia, prolonged QT interval and Osborn wave (Figure 5). Other causes of hypothermia such as hypothyroidism and hypoadrenalism were ruled out with normal thyroid function tests and fasting serum cortisol levels. Warm saline infusions and intermittent rewarming were given at the time of hypothermia. She developed intractable seizures and autonomic instability and succumbed to her illness after 2 weeks of hospital care.

Written informed consent was obtained from the parents of the patient for the publication of this report and accompanying images.

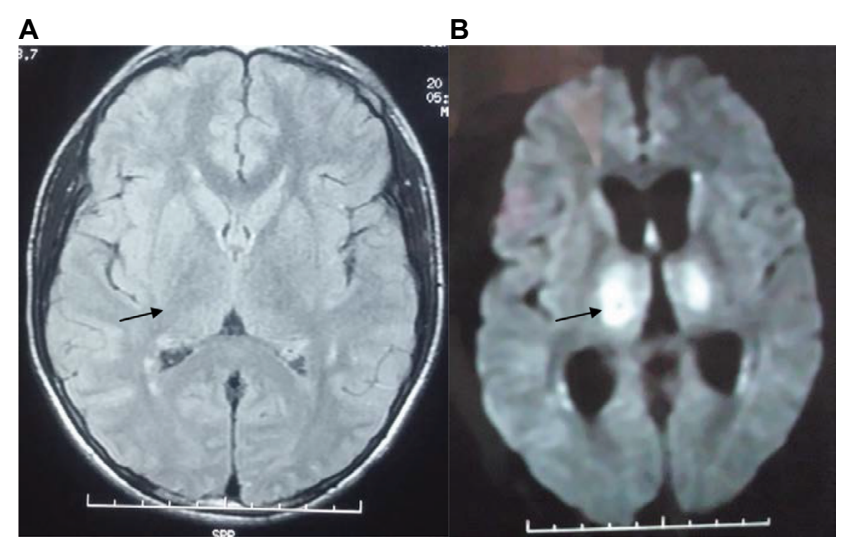

Figure $3 \mathrm{MRI}$ showing thalamic hyperintensities (arrows). T2 weighted image (A); diffusion weighted image (B).

Abbreviation: MRI, magnetic resonance imaging.

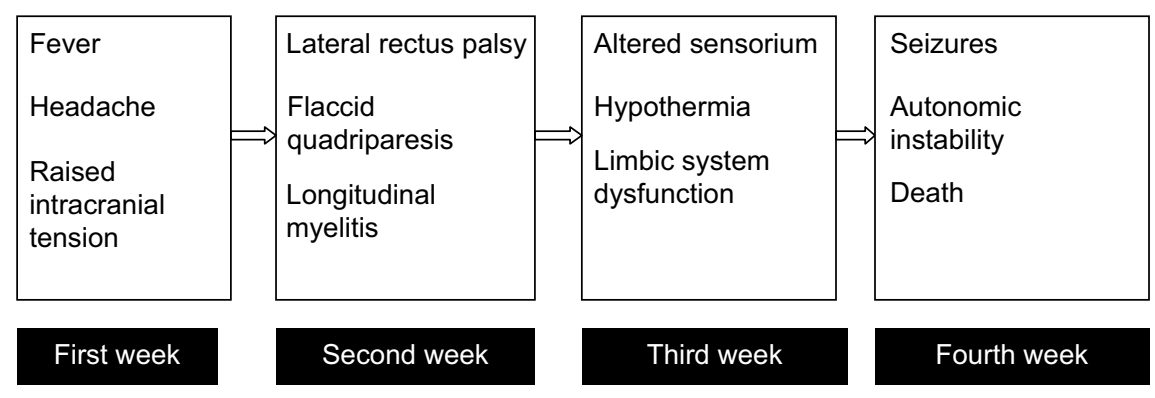

Figure 2 Clinical course of the patient. 


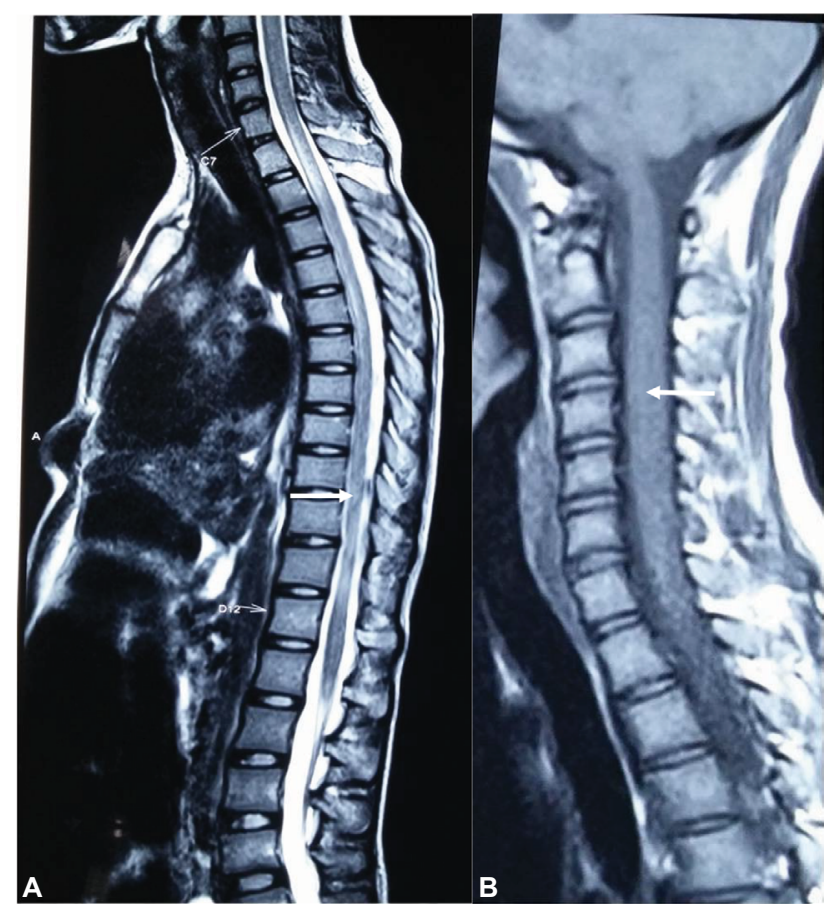

Figure 4 Longitudinal myelitis extending caudally upto $\mathrm{LI}$ and rostrally upto pons. T2 weighted image (A); TI weighted image (B). The white arrows show white matter hyperintensities.

\section{Discussion}

$\mathrm{JE}$ is one of the important causes of viral encephalitis in the Asian population. It is caused by JE virus, which belongs to Flaviviridae, and is related to its Western counter parts Saint Louis encephalitis virus and West Nile virus. ${ }^{1}$ Mosquitoes of the subgroup, especially Culex tritaeniorhynchus, are the major vectors of JE virus. Pigs and migrating birds such as egrets are the amplifier hosts. The risk of transmission is highest in the agricultural and rural areas. Symptomatic neurological diseases occur in $<1 \%$ of the individuals, with most of them presenting as nonspecific febrile illness. ${ }^{2}$ In neuroinvasive disease, initial nonspecific symptoms of fever and headache are followed by the development of focal neurological deficits, seizures, disturbances of higher mental functions and coma in very severe cases. ${ }^{3}$ Some patients develop psychosis-like symptoms, whereas in others, extrapyramidal involvement results in movement disorders. ${ }^{4}$

A rare presentation includes flaccid paralysis mimicking acute poliomyelitis due to involvement of anterior horn cells. ${ }^{5}$ Poliomyelitis presents usually as acute transverse myelitis, whereas in our case, the onset and progression of weakness was asymmetrical with MRI, showing the presence of longitudinal myelitis extending upto pons. Such a presentation is very rare in JE, and there are scarce reports.

The limbic system consists of a complex network in brain concerned with instinct and mood and includes the 1) diencephalic structures (hypothalamus, anterior nuclei of thalamus and mammillary bodies); 2) cortical areas (orbitofrontal cortex, entorhinal cortex, piriform cortex, hippocampus and the fornix) and 3) subcortical areas (septal nuclei, amygdala and nucleus accumbens). ${ }^{6}$ The etiology of limbic encephalitis falls into two categories: infectious encephalitis and autoimmune encephalitis. The involvement of limbic system in viral encephalitis is most commonly described in herpes encephalitis and is also prototypical in many autoimmune encephalitides. There are very less data on the involvement of limbic system in JE. The involvement of thalamic regions is described in many cases of JE, the mechanism of which is still to be elucidated. Hypothalamic dysfunction as a part of limbic encephalitis results in loss of temperature regulation and hypothermia as seen in our patient. It can also result in autonomic instability with cardiac arrhythmias and endocrine abnormalities such as diabetes insipidus and hypothyroidism. ${ }^{7}$ The cause of death in our patient was due to autonomic instability with wide fluctuation in vital signs. Other causes of hypothermia include hypothyroidism, adrenal insufficiency, sepsis, neuromuscular disease, malnutrition, thiamine deficiency, ethanol abuse and carbon monoxide intoxication. ${ }^{8}$

The complications of hypothermia include lactic acidosis, rhabdomyolysis, bleeding diathesis and infection. Electrocardiographic abnormalities include bradyarrhythmias, Osborn wave, prolonged QT interval and ventricular

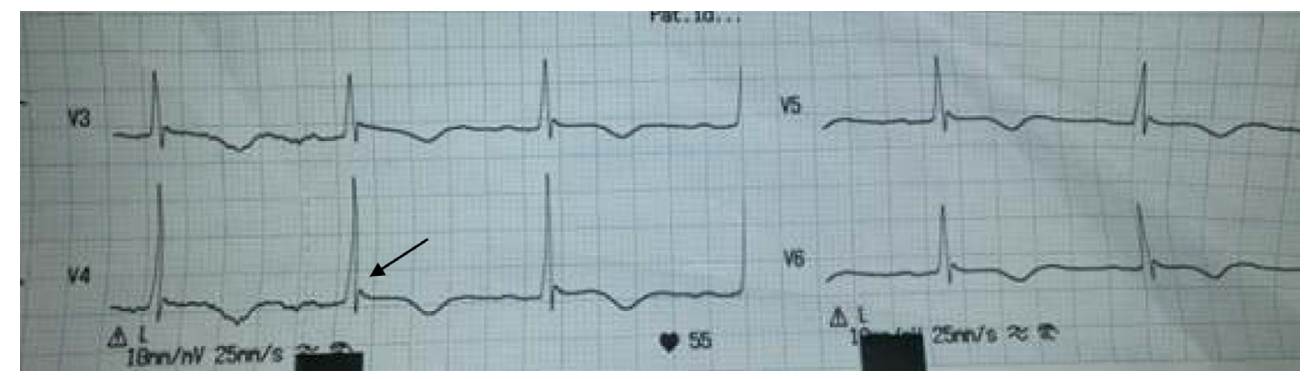

Figure 5 ECG showing bradycardia, prolonged QT interval and Osborn Wave (arrow). Abbreviation: ECG, electrocardiography. 
ectopics culminating in ventricular fibrillation in severe hypothermia. ${ }^{9}$ Our patient also had the abovementioned findings except for ventricular fibrillation. The Osborn wave ( $\mathrm{J}$ wave) is a positive deflection at the $\mathrm{J}$ point (negative in lead aVR and V1). Mild hypothermia is treated with external rewarming and warm crystalloid infusions, whereas severe hypothermia warrants therapy with extracorporeal warming solutions and cardiopulmonary bypass. ${ }^{10}$ External rewarming may be passive or active. Passive external rewarming comprises insulating the patient in a warm environment. The patient is rewarmed at a rate of $1.1-4.4^{\circ} \mathrm{F} /$ hour. Active rewarming is needed when the core temperature is $<90^{\circ} \mathrm{F}$. External heat exchange pads, radiant heat sources and hot packs are modes of active external rewarming. Active core rewarming is achieved by rewarming the airway through humidified oxygen and by delivering heated crystalloids. Closed thoracic lavage and peritoneal lavage are efficient modes of delivering heat when the patient is severely hypothermic. Extracorporeal rewarming of blood is needed along with active rewarming in severe hypothermia. The different techniques include continuous venovenous rewarming, continuous arteriovenous rewarming, hemodialysis and extracorporeal membrane oxygenation. The rate of rewarming is $2-3^{\circ} \mathrm{F} /$ hour. Cardiopulmonary bypass with circulatory support is the only option if the extremities are frozen and in instances with a significant tissue destruction.

The most classical MRI finding in JE is T2-weighted fluid attenuated and inversion recovery hyperintense lesions in bilateral thalami. On T1-weighted images, these lesions are hypointense. The lesions also undergo hemorrhagic transformation. The cytotoxic edema leads to restricted diffusion in diffusion MRI. ${ }^{11}$ The differential diagnosis of these findings includes deep cerebral vein thrombosis of thalamic veins and thalamic infarcts due to basilar artery occlusion.

$\mathrm{JE}$ is diagnosed serologically by the detection of specific IgM antibodies either in CSF (appears by 5-8 days) or in serum (appears by $8-9$ days). ${ }^{12}$ Other tests for virus isolation include detection of viral RNA by nucleic acid amplification test and reverse transcription polymerase chain reaction (RT-PCR), but the results are often negative as viremia is transient. ${ }^{13}$

Treatment of JE is supportive, which includes treatment of raised intracranial pressure and seizures. Neither antiviral nor corticosteroids have shown any efficacy. ${ }^{14}$ Osmotic diuretics are used to reduce intracranial tension. The modes of prevention of JE include vaccination and vector control measures. Two vaccines are available for clinical use: an inactivated mouse brain-derived vaccine obtained from Nakayama JE virus strain and an inactivated Vero cell culture-derived vaccine obtained by propagating SA-14-14-2 JE virus strains in Vero cells. Two doses are administered intramuscularly 28 days apart. Vaccination is indicated for travelers to endemic areas in Asia. The newer DNA vaccine is undergoing clinical trial.

\section{Conclusion}

JE should be among the most important differential diagnoses of patients presenting with fever and altered sensorium in endemic regions. Although it presents usually as seizures and with extrapyramidal symptoms, unusual presentations such as flaccid paralysis and limbic system involvement, as in our case, should be kept in mind. Neuroimaging and CSF analysis are important aids to early diagnosis.

\section{Disclosure}

The authors report no conflicts of interest in this work.

\section{References}

1. Mackenzie JS, Williams DT, Smith DW. Japanese encephalitis virus: the geographic distribution, incidence, and spread of a virus with a propensity to emerge in new areas. In: Tabor E, editor. Emerging Viruses in Human Populations. Amsterdam: Elsevier; 2006;16:201-268.

2. Solomon T, Dung NM, Kneen R, et al. Seizures and raised intracranial pressure in Vietnamese patients with Japanese encephalitis. Brain. 2002; 125(pt 5):1084.

3. Solomon T, Dung NM, Kneen R, et al. Japanese encephalitis. J Neurol Neurosurg Psychiatry. 2000;68(4):405.

4. Kuwayama M, Ito M, Takao S, et al. Japanese encephalitis virus in meningitis patients, Japan. Emerg Infect Dis. 2005;11(3):471.

5. Chung CC, Lee SS, Chen YS, et al. Acute flaccid paralysis as an unusual presenting symptom of Japanese encephalitis: a case report and review of the literature. Infection. 2007;35(1):30.

6. Morgane PJ, Galler JR, Mokler DJ. A review of systems and networks of the limbic forebrain/limbic midbrain. Prog Neurobiol. 2005;75(2):143-160.

7. Wang XL, Lu JM, Yang LJ, et al. A case of relapsed autoimmune hypothalamitis successfully treated with methylprednisolone and azathioprine. Neuro Endocrinol Lett. 2008;29(6):874-876.

8. Lee-Chiong TL Jr, Stitt JT. Disorders of temperature regulation. Compr Ther. 1995;21(12):697.

9. Olgers TJ, Ubels FL. The ECG in hypothermia: Osborn waves. Neth $J$ Med. 2006;64(350):353.

10. Kempainen RR, Brunette DD. The evaluation and management of accidental hypothermia. Respir Care. 2004;49(2):192.

11. Lim CCT, Sitoh YY, Hui F, et al. Nipah viral encephalitis or Japanese encephalitis MR findings in a new zoonotic disease. AJNR Am J Neuroradiol. 2000;21(3):455-461.

12. Chanama S, Sukprasert W, Sa-ngasang A, et al. Detection of Japanese encephalitis (JE) virus-specific IgM in cerebrospinal fluid and serum samples from JE patients. Jpn J Infect Dis. 2005;58(5):294.

13. Swami R, Ratho RK, Mishra B, Singh MP. Usefulness of RT-PCR for the diagnosis of Japanese encephalitis in clinical samples. Scand J Infect Dis. 2008;40(10):815.

14. Kumar R, Tripathi P, Baranwal M, et al. Randomized, controlled trial of oral ribavirin for Japanese encephalitis in children in Uttar Pradesh, India. Clin Infect Dis. 2009;48(4):400. 
The International Journal of General Medicine is an international, peer-reviewed open-access journal that focuses on general and internal medicine, pathogenesis, epidemiology, diagnosis, monitoring and treatment protocols. The journal is characterized by the rapid reporting of reviews, original research and clinical studies across all disease areas.
The manuscript management system is completely online and includes a very quick and fair peer-review system, which is all easy to use. Visit http://www.dovepress.com/testimonials.php to read real quotes from published authors. 